\title{
Iterative MIMO Detection for Rank-Deficient Systems
}

\author{
A. Wolfgang, J. Akhtman, S. Chen, and L. Hanzo
}

\begin{abstract}
In this letter, a novel iterative multiple-input multiple-output (MIM)O detector reminiscent of sphere decoding (SD) is presented. Its main benefit is that in contrast to other softinput soft-output (SISO) SD algorithms presented in the literature, the proposed iterative detector is capable of performing well in so-called overloaded systems, where the number of transmit antennas is significantly higher than the number of receive antennas, and hence, the channel's covariance matrix is rank-deficient. Furthermore, the proposed detector is guaranteed to achieve the performance of the max-log detector without the necessity of choosing a specific SD radius or an SD candidate list for generating soft-outputs. For example, when receiving signals from four transmit antennas with the aid of only two receive antennas, the proposed detector is capable of approaching the channel capacity limit within about 2 dBs.
\end{abstract}

Index Terms-Iterative detection, multiple-input multipleoutput (MIMO), sphere decoding (SD).

\section{INTRODUCTION}

I TERATIVE detection in the context of multiple-input multiple-output (MIMO) systems has been investigated using different soft-input soft-output (SISO) detector designs such as the minimum mean-squared error (MMSE) SISO detector based on [1], which is particularly attractive owing to its low computational complexity. In systems where the number of transmitters to be detected is not significantly higher than the number of received signals, the MMSE detector is capable of approaching the performance of the optimum maximum a posteriori (MAP) detector at the cost of an increased number of iterations.

Approximate MAP detectors employing sphere decoding (SD) have been discussed in [2], where the so-called list sphere decoder (LSD) was introduced. However, the system considered in [2] employs an equal number of receive and transmit antenna elements (AEs), since the detector tends to rely on finding the least-square (LS) estimate of the transmitted signal, which implies that the covariance matrix of the channel is nonsingular.

Sphere decoders have been proposed for rank-deficient systems, for example, in [3]-[5], but none of these contributions considers SISO-aided iterative detection. It is worth mentioning that it would be possible to employ any of the SDs designed for rank-deficient systems in the context of the LSD proposed in [2] in order to obtain a SISO SD for overloaded scenarios. However,

Manuscript received February 18, 2006; revised April 21, 2006. This work was supported by the European Union under the auspices of the Phoenix and Newcom projects. The associate editor coordinating the review of this manuscript and approving it for publication was Prof. Richard J. Kozick.

The authors are with the School of Electronics and Computer Science, University of Southampton, Southampton, SO17 1BJ, U.K. (e-mail: aw03r@ecs. soton.ac.uk; yja02r@ecs.soton.ac.uk; sqc@ecs.soton.ac.uk; lh@ecs.soton.ac. uk).

Digital Object Identifier 10.1109/LSP.2006.879453 the resultant SD would not necessarily find the max-log solution, and the performance of the detector would strongly depend on the size of the candidate list considered by the SD. If the processing of the soft information would be directly included in the evaluation of a conventional SD rather than choosing a candidate list, the appropriate choice of the SD radius would impose a challenge.

By contrast, the SISO reduced-complexity max-log (RML) algorithm presented in this contribution is a true SISO detector, which does not impose any of the aforementioned constraints on the system and thus may be invoked in scenarios, where the number of transmit AEs is significantly higher than the number of receive AEs. It directly includes the processing of soft-information in the algorithm without the necessity of creating a candidate list or choosing an SD radius. The algorithm constitutes a logical evolution of the orthogonal hierarchical recursive search algorithm (OHRSA) of [6] proposed for maximumlikelihood (ML) MIMO detection in multicarrier systems. The outline of this letter is as follows. In Section II, the system model is introduced, while the advocated algorithm is derived in Section III. In Section IV, our simulation results are presented, and in Section V, we offer our conclusions.

\section{SySTEM MODEL}

In order to keep the notation simple, we consider a narrowband single-user MIMO system characterized by an $M$-element transmit antenna array and an $N$-element receive antenna array. The equations derived are also valid if multiple MIMO users are considered in a synchronous uplink scenario. The single user's transmitter channel encodes the input bit-stream at a code-rate $R$, interleaves the encoded bits, modulates the signal, and maps it to the $M$ different transmit AEs. The modulated symbols are transmitted over a narrowband channel characterized by the channel coefficients $h_{n m}$, which represent the complex-valued channel between the $m$ th transmit and the $n$th receiver AE. Denoting the symbol transmitted by AE $m$ as $s_{m}$, the output signal of the $n$th receive $\mathrm{AE}$ at time instant $k$ can be written as

$$
x_{n}(k)=\sum_{m=1}^{M} h_{n m} s_{m}(k)+\eta(k)
$$

where $\eta(k)$ is the complex-valued additive white Gaussian noise (AWGN) having a variance of $E\left[\left|\eta_{n}(k)\right|^{2}\right]=2 \sigma^{2}$.

Assuming unity transmit power per transmit $\mathrm{AE}$, the resultant $E_{b} / N_{0}$ is given as

$$
\frac{E_{b}}{N_{0}}=\frac{\sum_{n=1}^{N} \sum_{m=1}^{M} E\left[\left|h_{n m}\right|^{2}\right]}{R \log _{2}(\mathcal{M}) M N 2 \sigma^{2}}
$$

where $\mathcal{M}$ is the number of modulation levels. 


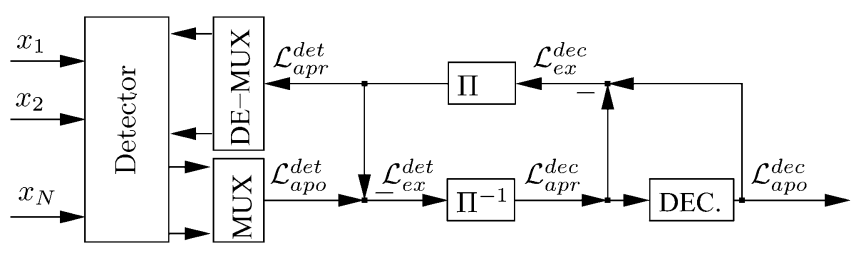

Fig. 1. MIMO receiver model employing iterative detection.

Under the assumption of perfectly synchronized transmitters, the relation between the signal transmitted and the channel's output is described by an $(N \times M)$-dimensional matrix $\mathbf{H}$, where the $(n, m)$ th element of the matrix is given by $h_{n m}$. The $N$-element channel output vector $\mathbf{x}(k)$ can be expressed as

$$
\mathbf{x}(k)=\mathbf{H}(k) \mathbf{s}(k)+\boldsymbol{\eta}(k)
$$

where $\mathbf{s}(k)=\left[s_{1}(k), \ldots, s_{M}(k)\right]^{T}$ is a column vector containing the transmitted symbols, and the AWGN vector is given by $\boldsymbol{\eta}(k)=\left[\eta_{1}(k), \ldots \eta_{N}(k)\right]^{T}$. For notational simplicity, the time-index $k$ will be dropped, where this is possible without ambiguity.

The iterative detection scheme considered is depicted in Fig. 1, where $\mathcal{L}$ represents the log-likelihood ratios (LLRs), which are used to pass information between the different receiver components. The superscript det indicates the detector, while dec indicates the channel decoder. The subscripts $a p r$, $e x$, and apo indicate a priori, extrinsic, and a posteriori LLRs, respectively. For a detailed description of iterative equalization, the interested readers are referred to [7].

\section{SISO MAX-LOG DETECTOR}

In this section, the SISO max-log algorithm designed for the detection of the transmitted signal is highlighted [8]. The max-log approximation of the desired LLRs required for the detection of the transmitted signal outlined in (1) can be formulated as

$$
\mathcal{L}_{\text {apo }}^{\operatorname{det}}\left(s_{m}\right) \approx \log \left(\frac{\max _{\breve{\mathbf{s}} \in \mathcal{S}^{(+)}} P(\breve{\mathbf{s}}) p(\breve{\mathbf{s}} \mid \mathbf{x})}{\max _{\breve{\mathbf{s}} \in \mathcal{S}^{(-)}} P(\breve{\mathbf{s}}) p(\breve{\mathbf{s}} \mid \mathbf{x})}\right)
$$

where $\mathcal{S}^{( \pm)}$is the set of potentially transmitted vectors $\breve{\mathbf{s}}, 1 \leq$ $m \leq M$ associated with $\breve{s}_{m}= \pm 1$, and $P(\breve{\mathbf{s}})$ is the a priori probability of the given hypothetical signal vector being considered, which may be obtained from the a priori bit LLRs $\mathcal{L}_{\text {apr }}^{\text {dec }}\left(s_{m}\right)$ as outlined in [2], assuming independence of the individual bits. Equation (4) may now be rewritten as

$$
\begin{aligned}
\mathcal{L}_{\text {apo }}^{\text {det }}\left(s_{m}\right)= & J_{\min }\left(\breve{s}_{m}=-1\right)-J_{\min }\left(\breve{s}_{m}=+1\right) \\
= & \min _{\breve{\mathbf{s}} \in \mathcal{S}(-)}\left\{-\log (P(\breve{\mathbf{s}}))+\frac{|\mathbf{x}-\mathbf{H} \breve{s}|^{2}}{2 \sigma^{2}}\right\} \\
& -\min _{\breve{\mathbf{s}} \in \mathcal{S}^{(+)}}\left\{-\log (P(\breve{\mathbf{s}}))+\frac{\|\mathbf{x}-\mathbf{H} \breve{\mathbf{s}}\|^{2}}{2 \sigma^{2}}\right\}
\end{aligned}
$$

where $J$ represents the cost-function to be optimized, and $J_{\min }\left(\breve{s}_{i}=j\right)$ represents the minimum cost-function value under the constraint of $\breve{s}_{i}=j$. The algorithm presented in this section has the capability of solving the optimization problem associated with $J_{\min }\left(\breve{s}_{m}= \pm 1\right)$ at a moderate computational cost. Let us assume for the derivation of the algorithm that both the CIR matrix $\mathbf{H}$ as well as the noise are real-valued and that the transmitted signal is binary phase shift keying (BPSK) modulated. It was shown in [6] that the solution to the problem defined by (5) is identical to solving

$$
J_{\min }\left(\breve{s}_{m}=+1\right)=C+\min _{\breve{\mathbf{s}} \in \mathcal{S}(+)} \frac{\left|\mathbf{U}\left(\breve{\mathbf{s}}-\hat{\mathbf{s}}_{\mathrm{MMSE}}\right)\right|^{2}}{2 \sigma^{2}}-\log (P(\breve{\mathbf{s}}))
$$

where the upper triangular matrix $\mathbf{U}$ is defined by

$$
\mathbf{U}^{H} \mathbf{U}=\mathbf{H}^{H} \mathbf{H}+\sigma^{2} \mathbf{I}_{M}
$$

while

$$
\hat{\mathbf{s}}_{\mathrm{MMSE}}=\left(\mathbf{H}^{H} \mathbf{H}+\sigma^{2} \mathbf{I}_{M}\right)^{-1} \mathbf{H}^{H} \mathbf{x}
$$

where $\mathbf{I}_{M}$ is the $(M \times M)$-dimensional identity matrix. The constant $C$ is identical for both $J_{\min }\left(\breve{s}_{m}=+1\right)$ as well as $J_{\min }\left(\breve{s}_{m}=-1\right)$, and hence, it can be dropped from the evaluation of the a posteriori LLRs given in (5).

Exploiting the fact that the matrix $\mathbf{U}$ has an upper triangular structure, it can be shown that the objective function used for the detection of the transmitted symbol vector $\breve{s}$ may be written as [8]

$$
\begin{aligned}
J(\breve{\mathbf{s}}) & =\frac{\left|\mathbf{U}\left(\breve{\mathbf{s}}-\hat{\mathbf{s}}_{\mathrm{MMSE}}\right)\right|^{2}}{2 \sigma^{2}}-\log (P(\breve{\mathbf{s}})) \\
& =\frac{\left(\breve{\mathbf{s}}-\hat{\mathbf{s}}_{\mathrm{MMSE}}\right)^{H} \mathbf{U}^{H} \mathbf{U}\left(\breve{\mathbf{s}}-\hat{\mathbf{s}}_{\mathrm{MMSE}}\right)}{2 \sigma^{2}}-\log (P(\breve{\mathbf{s}})) \\
& =\sum_{i=1}^{M} \frac{\left|\sum_{j=i}^{M} u_{i j}\left(\breve{s}_{j}-\hat{s}_{j, \mathrm{MMSE}}\right)\right|^{2}}{2 \sigma^{2}}-\log \left(P\left(\breve{s}_{i}\right)\right) \\
& =\sum_{i=1}^{M} \phi_{i}\left(\breve{\mathbf{s}}_{i}\right)
\end{aligned}
$$

where $u_{i j}$ is the $(i, j)$ th element of $\mathbf{U}, \breve{\mathbf{s}}_{i}=\left[\breve{s}_{i}, \ldots, \breve{s}_{N_{s}}\right]^{T}$, and $\phi_{i}\left(\breve{\mathbf{s}}_{i}\right)$ may be expressed as [8]

$$
\phi_{i}\left(\breve{\mathbf{s}}_{i}\right)=\frac{\left.\mid u_{i i}\left(\breve{s}_{i}-\hat{s}_{i, \mathrm{MMSE}}\right)+a_{i}\right)\left.\right|^{2}}{2 \sigma^{2}}-\log \left(P\left(\breve{s}_{i}\right)\right)
$$

where

$$
a_{i}=\sum_{j=i+1}^{M} u_{i j}\left(\breve{s}_{j}-\hat{s}_{j, \mathrm{MMSE}}\right) .
$$

The term $a_{i}$ of (10) is independent of the specific symbol's value of $\breve{s}_{i}$. The cost function given in (9) may now be rewritten in a recursive manner as

$$
J_{i}\left(\breve{\mathbf{s}}_{i}\right)=J_{i+1}\left(\breve{\mathbf{s}}_{i+1}\right)+\phi_{i}\left(\breve{\mathbf{s}}_{i}\right), \quad i=N_{s}-1, \ldots 1
$$

where we have $J_{M}\left(\breve{\mathbf{s}}_{N_{s}}\right)=\left|u_{N_{s} N_{s}}\left(\breve{s}_{N_{s}}-\hat{s}_{N_{s}, \mathrm{MMSE}}\right)\right|^{2}$. The cost function has the essential property that [8]

$$
\begin{aligned}
J(\breve{\mathbf{s}}) & =J_{1}\left(\breve{\mathbf{s}}_{1}\right)>J_{2}\left(\breve{\mathbf{s}}_{2}\right)>\cdots>J_{N_{s}}\left(\breve{\mathbf{s}}_{N_{s}}\right) \\
& =J_{N_{s}}\left(\breve{s}_{N_{s}}\right)>0 .
\end{aligned}
$$

Based on (9)-(13), a low-complexity search technique employing a tree-search algorithm, which is outlined in detail in [9], [6], and [8], may be employed. A further property of 
the recursive cost-function imposed by the available a priori information is that we have

$$
\begin{aligned}
J_{M}(\breve{\mathbf{s}})> & J_{i}\left(\breve{\mathbf{s}}_{i}\right) \\
& -\sum_{j=1}^{i+1} \max \left\{\log \left(P\left(s_{j}=-1\right)\right), \quad \log \left(P\left(s_{j}=+1\right)\right)\right\}
\end{aligned}
$$

which might be exploited by the search tree algorithm in order to improve its convergence rate.

\section{Algorithm 1 Max-Log Metric Calculation $(\mathrm{x}, \mathrm{H})$}

$J_{\mathrm{ML}}=10^{10}$ (Value higher than true $J_{M L}$ )

$J_{\min }\left(\breve{s}_{i}= \pm 1\right):=$ a constraint $>J_{M L}$ for $1 \leq i \leq M$

for $i=1$ to $M$ do

$$
\begin{aligned}
& \text { for } j \in\{-1,1\} \\
& \text { if } J_{\min }\left(\breve{s}_{i}=j\right)>J_{\mathrm{ML}} \text { then } \\
& \text { calculate } J_{\min }\left(\breve{s}_{i}=j\right) \text { evaluating } \\
& \text { for } p=1 \text { to } M \text { do } \\
& J_{\min }\left(\breve{s}_{p}=\breve{s}_{p \mid \breve{s}_{i}=j}\right)= \\
& \left.\min _{\breve{s}_{p \mid \breve{s}_{i}=j}\left\{J _ { \operatorname { m i n } } \left(\breve{s}_{p}=\right.\right.} J_{\min }\left(\breve{s}_{i}=j\right)\right\}
\end{aligned}
$$$$
\text { calculate } J_{\min }\left(\breve{s}_{i}=j\right) \text { evaluating search-tree }
$$

end for

$$
J_{\mathrm{ML}}=\min \left\{J_{\mathrm{ML}}, J_{\min }\left(\breve{s}_{i}=j\right)\right\}
$$

end if

end for

\section{end for}

The search tree algorithm is guaranteed to find the ML solution $\mathbf{s}_{\mathrm{ML}}$ satisfying the cost-function value $J_{\mathrm{ML}}=\min \left(J_{\mathrm{min}}\right)$. This constitutes an effective way of calculating the $2 M$ costfunction values required for obtaining the desired $M$ number of LLRs. The proposed method is summarized in Algorithm 1. The variables used are

- $J_{\min }\left(\breve{s}_{i}=j\right)$ : minimum cost-function value under the constraint $\breve{s}_{i}=j$;

- $\breve{s}_{p \mid \breve{s}_{i}=j}$ : symbol value of $\breve{s}_{p}$ associated with $J_{\min }\left(\breve{s}_{i}=j\right)$.

The proposed algorithm requires $M+1$ search tree passes for the calculation of the $2 M$ cost-function values. The algorithm exploits the fact that the cost-function value $J_{\mathrm{ML}}$ associated with the ML solution is the lowest possible cost-function value and therefore already provides the first $N_{s}$ necessary values $J_{\min }\left(\breve{s}_{i}=s_{i, \mathrm{ML}}\right)=J_{\mathrm{ML}}$. The algorithm may also readily be used for the detection of higher order modulation schemes by representing a complex-valued symbol as a string of independent bits, as outlined in [8]. The algorithm presented in this section extends the original hard-output OHRSA of [8] to a SISO algorithm, which may be employed in iterative systems. Furthermore, an effective algorithm for calculating the required $2 M$ cost-function values has been presented.
TABLE I

Serially Concatenated Channel Codes Used in OUR Simulations. Code 3 Was a Conventional RSC Code Without a Rate-1 Precoder

\begin{tabular}{|c|c|c|}
\hline & Rate-1 Code & RSC Code with constraint length $K$ \\
\hline Code 1 & $D /(1+D)$ & {$[56], K=3$} \\
\hline Code 2 & $D /(1+D)$ & {$[36], K=3$} \\
\hline Code 3 & none & {$[47], K=3$} \\
\hline
\end{tabular}

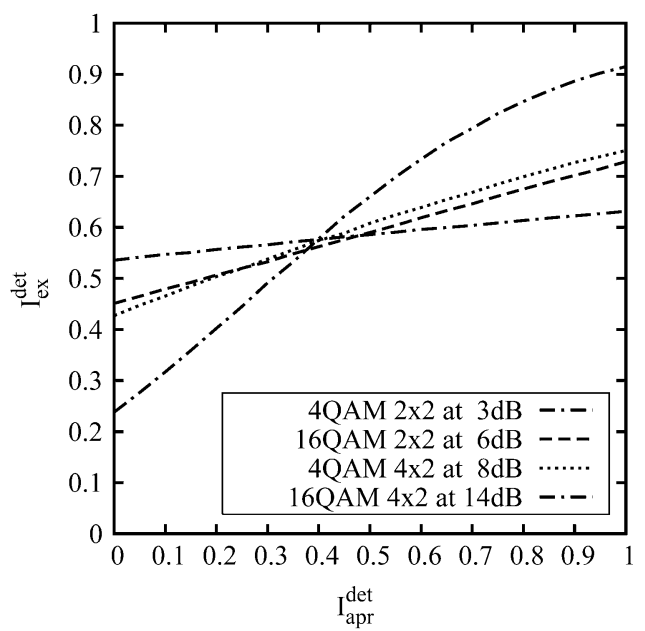

Fig. 2. Two-dimensional EXIT chart [10] for a MIMO system associated with $M=2$ or 4 transmit antennas and $N=2$ receive antennas at different $E_{b} / N_{0}$ values, as defined in (2) for a code-rate of $R=1 / 2$. The modulation schemes considered were 4QAM and 16QAM.

\section{RESULTS}

The performance of the RML detector has been evaluated considering both 4QAM as well as 16QAM signals and a receiver employing $N=2$ antennas for receiving from $M$ number of transmit AEs at different $E_{b} / N_{0} \cdot{ }^{1}$ The channel codes used are described in Table I. The random bit interleaver employed had a length of $2^{16}$ bits, and the fading process imposed by the channel was assumed to be ergodic and uncorrelated between the different AEs.

In Fig. 2, the proposed RML detector is characterized with the aid of EXtrinsic Information Transfer (EXIT) charts [10] for both $M=2$ as well as $M=4$ transmit AEs. It can be observed that for $4 \mathrm{QAM}$ signals as well as for the $(2 \times 2)$-antenna 16QAM scenario considered, the EXIT function is almost horizontal. ${ }^{2}$ As a benefit, in conjunction with a well-designed channel code, which matches this EXIT characteristic, the iterative detector has a low complexity, since it needs only a few detection iterations in order to converge. By contrast, the EXIT function associated with the $(4 \times 2)$-antenna overloaded 16QAM scenario shows a higher gradient, which implies that an open EXIT tunnel in conjunction with an appropriately designed channel code EXIT function can be readily created, but

\footnotetext{
${ }^{1}$ Plotting the EXIT functions for a fixed $E_{b} / N_{0}$ as defined in (2), rather than for a fixed signal-to-noise ratio (SNR) allows a fair comparison for different modulation schemes and code rates.

${ }^{2}$ In contrast to the typical channel code EXIT characteristics, the EXIT functions of iterative detectors generally do not reach the point of perfect convergence at $\left(I_{\mathrm{apr}}^{\mathrm{det}}, I_{\mathrm{ex}}^{\text {det }}\right)=(1,1)$.
} 


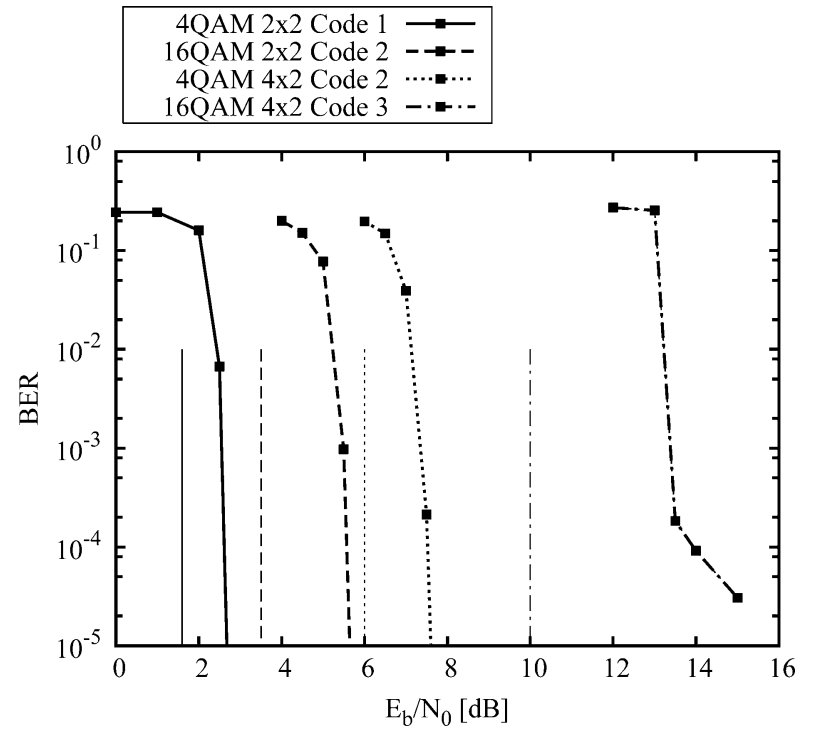

Fig. 3. BER versus $E_{b} / N_{0}$ for a MIMO system having $M=2$ or 4 transmit antennas and $N=2$ receive antennas and using 4QAM or 16QAM. The channel codes employed are the various serially concatenated codes of Table I.

iterating through this steeper tunnel may involve many detection iterations and thus a high computational complexity. Furthermore, it can be observed in Fig. 2 that the overloaded $(4 \times 2)$-antenna 4QAM and the non-overloaded $(2 \times 2)$-antenna 16QAM have similar EXIT functions, and they both support identical system throughputs of $4 \cdot 2=2 \cdot 4=8$ (bit/MIMO symbol). The 16QAM system, however, has a $2-\mathrm{dB} E_{b} / N_{0}$ advantage in comparison to the overloaded 4QAM system.

In order to achieve a performance close to the channel capacity bound, in most cases, it is insufficient to consider an iteratively detected serially concatenated RSC code and a SISO detector. In order to support communication close to the channel capacity, we therefore consider a channel code consisting of a rate- 1 code and a RSC code, which are separated by an interleaver in order to facilitate iterative channel decoding. In our simulations, these two codes were viewed as one code where the number of inner iterations between the two codes was chosen to be 10 .

In Fig. 3, the achievable BER versus $E_{b} / N_{0}$ performance of the RML detector benefiting from iterative detection is illustrated together with the associated capacity bounds represented by the vertical lines. It can be observed that the performance of all scenarios is less than $2 \mathrm{~dB}$ away from the channel capacity, except for the $(4 \times 2)$-antenna 16QAM scenario. This can be explained by the fact that for this specific scenario, the code's EXIT function and the RML detector's EXIT function match poorly, and thus, the detector fails to approach the capacity limit. Communication closer to the capacity limit could be supported using, for example, irregular codes specifically designed to match the shape of the EXIT functions [11], which is naturally achieved at the cost of a higher number of detector iterations.

The complexity of the proposed algorithm is summarized in Table II for different iteration indexes. The normalized computational complexity is quantified in terms of the number of real-valued additions plus multiplications required for the evaluation of the soft-metric of a single bit. Only the computational complexity imposed by the evaluation of the search-tree was
TABLE II

Computational Cost (REAL-VAlued AdDitions Plus Multiplications) REQUiRED FOR THE EVAlUation OF THE SEARCH-TREE IN ORDER TO DETERMine THE LLR VALUE OF S SINGLE BIT

\begin{tabular}{|l|c|c|c|c|}
\hline Iteration Index & 1 & 2 & 3 & 4 \\
\hline $2 \times 24 Q A M$ at $3 \mathrm{~dB}$ & $1.1 \cdot 10^{2}$ & $1.1 \cdot 10^{2}$ & 97 & nan \\
\hline $4 \times 24 Q A M$ at $8 \mathrm{~dB}$ & $7.0 \cdot 10^{2}$ & $6.1 \cdot 10^{2}$ & $4.9 \cdot 10^{2}$ & $3.9 \cdot 10^{2}$ \\
\hline $2 \times 216 \mathrm{QAM}$ at $6 \mathrm{~dB}$ & $6.8 \cdot 10^{2}$ & $5.7 \cdot 10^{2}$ & $5.0 \cdot 10^{2}$ & $4.3 \cdot 10^{2}$ \\
\hline $4 \times 216 \mathrm{QAM}$ at $14 \mathrm{~dB}$ & $2.0 \cdot 10^{4}$ & $1.8 \cdot 10^{4}$ & $1.6 \cdot 10^{4}$ & $1.5 \cdot 10^{4}$ \\
\hline
\end{tabular}

considered. The computational cost is given for the condition, when the detector operates in the waterfall region, where the computational complexity imposed by the detector is the highest.

\section{CONCLUSION}

In this letter, we have presented a novel RML SISO detector, which is capable of reliably operating in rank-deficient systems, which are also often referred to as overloaded systems. Furthermore, an algorithm has been presented, which allows the effective calculation of the desired LLR values. It was shown through simulations that if the EXIT functions of the channel code and the RML detector are matched properly, communication close to the capacity bound can be achieved.

\section{ACKNOWLEDGMENT}

The authors would like to thank their colleagues for the enlightenment gained within the Phoenix consortium.

\section{REFERENCES}

[1] X. Wang and H. V. Poor, "Iterative (turbo) soft interference cancellation and decoding for coded CDMA," IEEE Trans. Commun., vol. 47, no. 7, pp. 1046-1061, Jul. 1999.

[2] B. Hochwald and S. Ten Brink, "Achieving near-capacity on a multiple-antenna channel," IEEE Trans. Commun., vol. 51, no. 3, pp. 389-399, Mar. 2003.

[3] T. Cui and C. Tellambura, "An efficient generalized sphere decoder for rank-deficient MIMO systems," IEEE Commun. Lett., vol. 9, no. 5, pp. 423-425, May 2005.

[4] M. Damen, K. Abed-Meraim, and J.-C. Belfiore, "Generalised sphere decoder for asymmetrical space-time communication architecture," Electron. Lett., vol. 36, no. 2, pp. 166-167, 2000.

[5] Z. Yang, C. Liu, and J. He, "A new approach for fast generalized sphere decoding in MIMO systems," IEEE Signal Process. Lett., vol. 12, no. 1, pp. 41-44, Jan. 2005.

[6] J. Akhtman and L. Hanzo, "Optimized-Hierarchy RSA-Aided SpaceTime Processing," in OFDM and MC-CDMA Primer: A Primer by L. Hanzo and T. Keller. New York: John Wiley, 2005, pp. 259-292. [Online]. Available: http://www-mobile.ecs.soton.ac.uk/comms/ Books.htm.

[7] L. Hanzo, C. H. Wong, and M. S. Yee, Adaptive Wireless Transceivers: Turbo-Coded, Turbo-Equalized and Space-Time Coded TDMA, CDMA, and OFDM Systems. . Piscataway, NJ: IEEE Press/Wiley, Feb. 2002.

[8] J. Akhtman and L. Hanzo, "Reduced-complexity maximum-likelihood detection in multiple-antenna-aided multicarrier systems," in Proc. 5th Int. Workshop Multi-Carrier Spread Spectrum, Oberpfaffenhofen, Germany, 2005, to be published.

[9] D. Pham, K. R. Pattipati, P. K. Willet, and J. Luo, "An improved complex sphere decoder for V-BLAST Systems," IEEE Signal Process. Lett., vol. 11, no. 9, pp. 748-751, Sep. 2004.

[10] S. Ten Brink, "Convergence behavior of iteratively decoded parallel concatenated codes," IEEE Trans. Commun., vol. 49, no. 10, pp. 1727-1737, Oct. 2001.

[11] J. Wang, S. X. Ng, A. Wolfgang, L. L. Yang, S. Chen, and L. Hanzo, "Near-capacity three-stage MMSE turbo equalization using irregular convolutional codes," in Proc. 4th Int. Symp. Turbo Codes, Munich, Germany, Apr. 3-7, 2006. 